\title{
Study of transport parameters and defect states in thin film perovskites under different environments - air or vacuum - and after light-soaking
}

\author{
Christophe Longeaud ${ }^{1,2, *}$ \\ ${ }^{1}$ Group of Electrical Engineering from Paris, CNRS (UMR 8507), Pierre and Marie Curie University, Paris-Sud11 University, \\ 11 rue Joliot Curie, 91190 Gif sur Yvette, France \\ ${ }^{2}$ IPVF, Ile-de-France Photovoltaic Institute (IPVF), 30 Route Départementale 128, 91120 Palaiseau, France
}

Received: 15 October 2019 / Received in final form: 11 November 2019 / Accepted: 5 December 2019

\begin{abstract}
We present some advanced characterization techniques developed to investigate on the optoelectronic properties of thin film semiconductors and apply them to perovskite layers. These techniques are the steady state photocarrier grating (SSPG) and the Fourier transform photocurrent spectroscopy (FTPS). The SSPG was developed to study the ambipolar diffusion length of carriers and the FTPS was imagined to measure the variations of the below gap absorption coefficient with the light energy, giving information on the defect densities of the gap responsible for this absorption. The potentialities of these techniques are first detailed and then exemplified by their application to thin film perovskites. To study their stability, these films were exposed to different environments, air or vacuum, and in their as-deposited state or after light-soaking with heavy light. We find that the diffusion length and density of states are quite stable, even after light-soaking, and suggest that the degradation of devices exposed to 1 sun mainly comes from the evolution of the contacts instead of the perovkite itself.
\end{abstract}

Keywords: Transport properties / characterization / light-soaking / perovskite

\section{Introduction}

Since the first demonstration that perovskites could be used as absorbers in solar energy conversion devices [1], these latters have shown in few years the fastest increase of conversion efficiency ever recorded. However, though cells and modules are close to be found on the market, some critical issues remain to be solved before reaching the reliability of well mastered technologies such as that of crystalline silicon. Among these issues the stability of the modules, working in outdoor conditions, has to be improved. So far, some of the origins of these instabilities are assumed to be linked to (i) the degradation of the perovskite (PVK) absorber by an air component (oxygen, moisture, etc.) and (ii) the influence of heavy light under which all solar devices are supposed to work.

In this communication we investigate some of the optoelectronic properties, and their evolution under different stresses, of perovskite thin films by means of several experiments, which, to our knowledge, were not systematically applied to this material.

\footnotetext{
* e-mail: longeaud@geeps. centralesupelec.fr
}

Triple cations (MaFaCs) PVK thin films, $\sim 600 \mathrm{~nm}$ thick, have been deposited onto glass substrates by spincoating and fitted with gold electrodes $1 \mathrm{~cm}$ long and 1.5-2 $\mathrm{mm}$ apart.

To study the transport properties of these samples we have used the Steady State Photo Conductivity (SSPC) technique and the Steady States Photocarrier Grating (SSPG) technique [2]. This last technique has been largely improved to automatically perform measurements within a few minutes [3] to follow the fast evolution of the ambipolar diffusion length when experimental conditions, e.g. samples in air or under vacuum, are modified. Details of this new set-up are given in Section 3. Besides, these techniques, SSPC and SSPG, have been applied before and after the films had been light-soaked under heavy light (approx. 1 sun).

In addition, on the same films, we have applied Fourier Transform Photocurrent Spectroscopy (FTPS) $[4,5]$ experiments to investigate on the absorption coefficient, and the density of states (DOS) at the origin of this latter, and their evolution with light-soaking. The bases and advantages of FTPS, compared to the constant photocurrent (CPM) technique [6], will be detailed in Section 3 . 
With all these techniques we have observed that exposures to air and heavy light were not systematically detrimental to the transport properties of the films. Indeed, for some samples the ambipolar diffusion length, estimated from SSPG, increased with exposure to air and was not much affected by light-soaking though a clear modification of the absorption coefficient was put into evidence by FTPS in this last case.

The SSPC measurements have also shown that, for some samples, the photocurrent was higher when the sample was under air than when the sample was under vacuum. However, light-soaking resulted in a marked decrease of the photocurrent. Thus, one may conclude that under light-soaking PVK films degrade leading to a decrease of the conversion efficiency when incorporated in a device.

Nevertheless, we shall show that the instability of the devices should be also sought in the possible evolution of the contacts and interfaces exposed to air or heavy light. Indeed, Glow Discharge Optical Emission Spectroscopy (GD-OES) [7] revealed that under light-soaking the gold of the contacts diffuses through the perovskite films. This diffusion of gold, already observed in devices [8], is probably highly detrimental to stable solar energy conversion.

\section{Experimental details}

In this study we have chosen to investigate on some of the properties of three cations PVK (methylamonium: Ma, formamidinium: $\mathrm{Fa}$ and $\mathrm{Cs}$ ). The thin films were prepared from a $\left(\mathrm{MA}_{0.166} \mathrm{FA}_{0.833}\right) \mathrm{Pb}\left(\mathrm{Br}_{0.166} \mathrm{I}_{0.833}\right)_{3}$ solution, also referred as MAFA, by dissolving $1.10 \mathrm{M} \mathrm{PbI} \mathrm{PL}_{2}$ (TCI Chemicals), 0.20 M PbBr 2 (Alfa Aesar), 1.00 M formamidinium iodide (FAI, Dyesol) and 0.20 M methyl ammonium bromide (MABr, Dyesol) in a mixture of DMSO:DMF $(4: 1$ in $\mathrm{v} / \mathrm{v})$ as solvent. To form the desired triple perovskite, also referred as MAFACs with formula $\mathrm{Cs}_{x} \mathrm{MA}_{0.17} \mathrm{FA}_{0.8331-x-y} \mathrm{~Pb}\left(\mathrm{Br}_{0.17} \mathrm{I}_{0.83}\right)_{3}$, the required quantity of $\mathrm{Cs}^{+}$was added from a stock precursor solution of CsI (Sigma Aldrich) $1.50 \mathrm{M}$ in DMSO. Perovskite films were deposited on glass by spin coating using a double plateau (2000 rpm to cast the precursor solution followed by $6000 \mathrm{rpm}$ to drip $100 \mu \mathrm{L}$ of chlorobenzene). Then, the samples were annealed at $100{ }^{\circ} \mathrm{C}$ during $30 \mathrm{~min}$. All the films studied below presented a concentration of cesium and an excess of $\mathrm{PbI}_{2}$ of $5-10 \%$.

To apply the different characterization techniques to this material, parallel gold electrodes, $\sim 1 \mathrm{~cm}$ long and 1.5-2 mm apart, were deposited either on the glass substrate or on the film after its deposition. When they were not under study the samples were kept under dark and primary vacuum $\left(10^{-1} \mathrm{mbar}\right)$. During measurements the samples were whether in air or in a cryostat dynamically pumped down to $10^{-6} \mathrm{mBar}$ and in their 'as-deposited' (AD) states or light-soaked (LS) during a given amount of time (more than $21 \mathrm{~h}$ and up to $45 \mathrm{~h}$ ).

To light-soak the films with heavy light we have used a high power white LED, the power density spectrum of which extends from 400 to $800 \mathrm{~nm}$ with a power density equivalent to that of an AM1.5G solar spectrum in the same spectral region. The fact that the LED light has no infrared component is not critical for the light-soaking process since according to the FTPS spectra the light is not, or very weakly, absorbed by the perovskite film for wavelengths larger than $800 \mathrm{~nm}$ (energy lower than $1.55 \mathrm{eV}$ ). The use of a high power LED has the advantage to preserve the film from heating, the light-soaking process being performed on a sample kept at room temperature or close to it.

\section{Advanced characterizations}

\subsection{The steady state photocarrier grating technique}

\subsubsection{The SSPG bases}

The steady state photocarrier grating technique was proposed in 1986 by D. Ritter et al. to investigate on the ambipolar diffusion length $L_{a m b}$ of photoconductive semi-insulating materials such as hydrogenated amorphous silicon (a-Si:H) and GaAs [2,9]. The basic principle of this technique is to illuminate the gap in between two parallel and ohmic electrodes deposited on a photoconductive sample with two laser beams interfering or not, the occurrence of interferences depending on the light polarization of the beams. If the polarization of the light is parallel to the electrodes an interference pattern is formed on and in the sample, the grating period $\Lambda$ being a function of the wavelength $\lambda$ and of the angle $\theta$ between the beams

$$
\Lambda=\frac{\lambda}{2 \sin (\theta / 2)} .
$$

At a coordinate $x$ on an axis perpendicular to the electrodes, taking its origin on one of them, the intensity of light $I(x)$ can be written

$$
I(x)=I_{1}+I_{2}+\gamma_{0} \sqrt{I_{1} I_{2}} \cos \left(\frac{2 \pi x}{\Lambda}\right),
$$

where $I_{1}$ and $I_{2}$ are the intensities of the light of each beam and $\gamma_{0}$ is a factor between 0 and unity taking account of the quality of the interferences (e.g. the contrast). If the polarizations of the beam are crossed, for instance by mean of a $\lambda / 2$ plate set in the path of one of the beams, the total intensity impinging the sample is constant and equal to $I(x)=I_{1}+I_{2}$.

The intensities of the two beams are not the same. One beam, has an intensity much higher than the other one, for instance $I_{1} \gg I_{2}$. This main beam fixes the state of the film and the other one, the probe beam, creates a small perturbation of the steady state of the film.

The sample is biased with a small DC voltage and one measures the excess of current due to the superimposition of the two beams in between the electrodes. To measure accurately this excess of current, the probe beam is chopped at a low frequency, say $10 \mathrm{~Hz}$, and, after amplification of this current by a current-voltage converter, it is recorded by means of a lock-in amplifier. 
Fixing the position of the $\lambda / 2$ plate so that the polarizations of the light for the two beams are parallel to the electrodes generates interferences on the sample and an excess of current, $I_{w i}$, is recorded. If the $\lambda / 2$ plate is rotated to cross the beam polarizations they are simply superimposing, giving an excess of current $I_{w o i}$. Ritter et al. have shown that the quantity $\beta=I_{w i} / I_{w o i}$ could be written as a function of the ambipolar diffusion length of the carriers

$$
\beta(\Lambda)=1-\frac{2 \gamma \gamma_{0}}{\left[1+\left(\frac{2 \pi L_{a m b}}{\Lambda}\right)^{2}\right]^{2}},
$$

where $\gamma$ is defined from the proportionality between the photocurrent $I_{p h}$ and the flux $F$ of the light, $I_{p h} \propto F^{\gamma}$. Though this product, $\phi=\gamma \gamma_{0}$, contains only limited information, a fit of the curve $\beta(\Lambda)$, with $\phi$ and $L_{a m b}$ as adjustable parameters, brings the $L_{a m b}$ value.

Another method to extract $L_{a m b}$ from the SSPG data is to modify equation (3) to end with

$$
\left(\frac{2 \pi}{\Lambda}\right)^{2}=\left(\frac{1}{L_{a m b}}\right)^{2}\left[\frac{\sqrt{\phi}}{\sqrt{(1-\beta) / 2}}-1\right],
$$

that leads to a linear variation of $(2 \pi / \Lambda)^{2}$ with $\frac{1}{\sqrt{(1-\beta) / 2}}$.

This formula was proposed by I. Balberg to underline the self consistency of the SSPG technique [10]. The advantage of such a plot is that any deviation from a straight line is easily seen and, in this case, the accuracy of the $L_{a m b}$ value can be questionable [11].

Since the pioneering work of Ritter et al. many publications were dedicated to a thorough study of the SSPG technique. It is impossible to quote all of them and the reader may refer to reviews of the SSPG technique and its application to several types of thin films proposed by R. Brüggemann [12,13].

The general trends coming out of these studies are that, to lead to reliable values of $L_{a m b}$, the SSPG experiment must be ideally performed:

- in the lifetime regime, that is under conditions for which the carrier lifetime is much higher than the dielectric relaxation time,

- with a 'low' electric field applied in between the electrodes,

- with a 'low' frequency to measure with a lock-in amplifier the contribution of the probe beam to the excess current.

Under these conditions the interferences impinging the sample create several arrays: arrays of free carriers and arrays of trapped carriers that eventually generate arrays of space charge and of internal electric field. If the grating spacing is small, these arrays are blurred and may even vanish under the influence of the diffusion of carriers. The excesses of current are thus almost equivalent leading to $\beta \approx 1$. On the other hand, with large grating periods a space charge array of minority carriers, resulting from the differences of the transport properties of the minority and majority carriers, establishes and the drift in between the electrodes of the majority carriers under the influence of the applied field is limited by the average internal field created by this space charge array. That is why the frequency of the chopper must be 'low', meaning low enough compared to the emission of carriers toward the bands so that a quasisteady-state is reached for the distribution of space charge. That is also why the applied field must be 'low' so that it does not excessively influence the space charge distribution modifying the internal field distribution. Of course these two 'low's ('low' frequency and 'low' field) are material dependent. For hydrogenated amorphous silicon (a-Si:H) we expect that a field of the order of $100 \mathrm{~V} / \mathrm{cm}$ and a frequency of the order of $10 \mathrm{~Hz}$ are convenient to perform reliable SSPG measurements [14]. It is these values that were also used to investigate on the perovskite ambipolar diffusion length.

An interesting point is that, in the SSPG technique, the limitation of the transport of the majority carriers by the minority carrier space charge is similar to the phenomenon of limitation of the majority carriers current by the space charge developed by the minority carriers in a device if these latter are not extracted properly from it. This point was confirmed during our long work on the study of a-Si:H thin films and devices for which we found that it is useless to make a device with a thin film with a poor $L_{a m b}$. On the other hand, if a device presents a weak energy conversion with an absorber having a large diffusion length, the reason may come from other issues as bad interfaces, resistive contacts, etc. Such a behavior was also observed with organic thin films and devices where the best devices were those incorporating films with the largest $L_{a m b}$ [15]. Therefore, as also underlined by Balberg [16], the SSPG technique seems to be an excellent technique to investigate on material transport properties before incorporating it into a device.

However, the major drawback of this experiment in its first designs is that the angle between the two beams has to be set manually for each value of $\Lambda$ [13]. Therefore, it is difficult to use it as a systematic characterization technique to study the influence of a deposition parameter on the quality of the prepared film because of the amount of time needed to obtain a $\beta(\Lambda)$ curve. That is why we have designed an automatic bench that covers a large range of $\Lambda$ within a few minutes.

\subsubsection{Description of an automatic SSPG bench}

This automatic SSPG bench has been described in details in [3]. We shall recall here the basic ideas of this realization, a schematic drawing of which is shown in Figure 1. A laser beam coming from a He-Ne laser, polarized perpendicularly to the plane of the figure, is split into two other beams. The probe beam is chopped and attenuated whereas a $\lambda / 2$ plate is set on the path of the main beam, to rotate the light polarization. The path of the main beam is chosen so that it reaches the sample perpendicularly to its plane in between the electrodes while the path of the probe beam is a little bit more complicated.

First, it is sent toward a mobile mirror that can be moved along a rail so that the beam is reflected toward one of the nine return mirrors. The angle between the main and the probe beam, when they reach the film, depends on the 


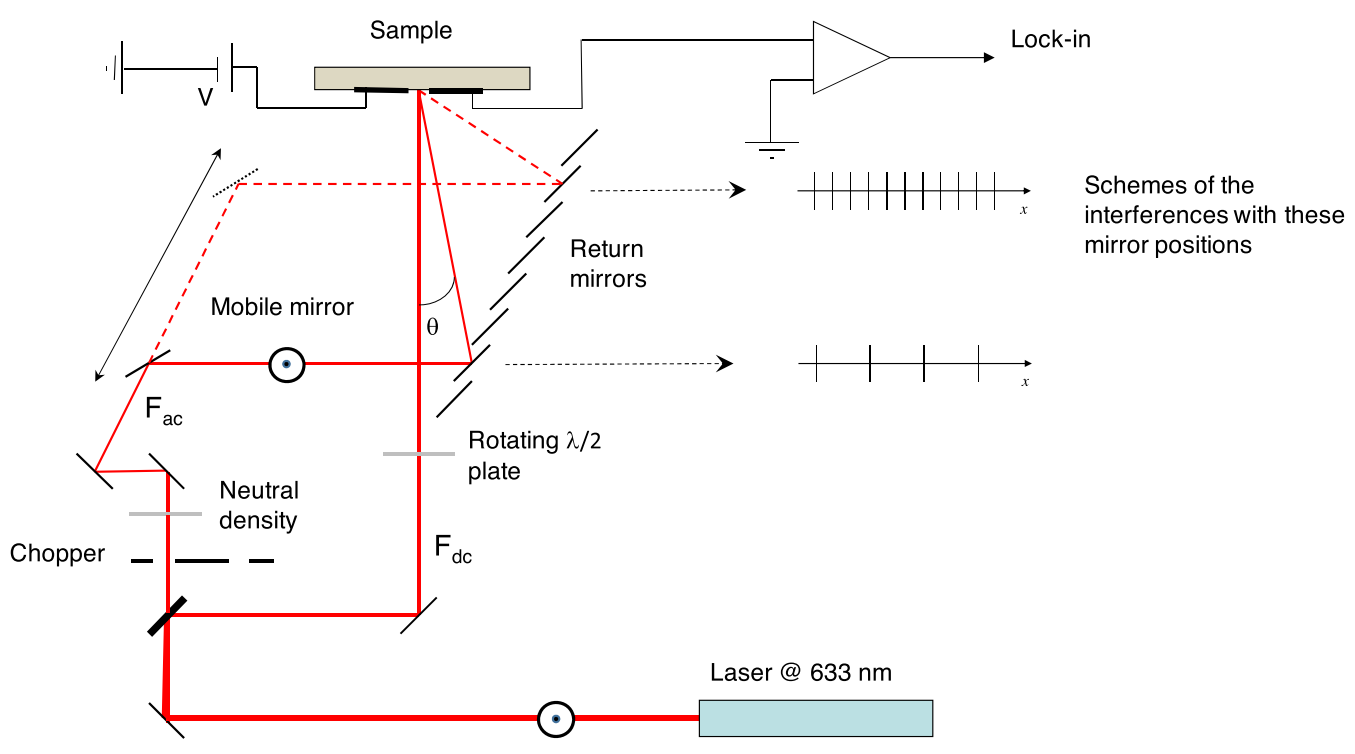

Fig. 1. Schematic diagram of an automatic SSPG bench.

chosen return mirror. To have a difference of the paths of the beams lower than the coherent length of the laser light, the rail is not parallel to the main beam. In addition, just before the mobile mirror, two mirrors are added to end with a path difference within $\pm 1 \mathrm{~cm}$.

The positions of the nine return mirrors are chosen so that the grating period $\Lambda$ varies in the range $\sim 1 \mu \mathrm{m}$, for the mirror close to the sample, to more than $20 \mu \mathrm{m}$ for the mirror the farthest from the sample. Note that, because of the path geometry chosen for the beams to illuminate the sample, the grating period is not defined according to equation (1) but rather by $\Lambda=\lambda / \sin (\theta)$.

This system saves the time needed to align manually the two beams for each $\Lambda$ before they reach the sample as in the case of a standard bench. All the alignments for each return mirror are tuned once and for further measurements on other samples the only adjustment is to fix the sample onto the sample holder. The position of the mobile mirror, the rotation of the $\lambda / 2$ plate and the acquisition of the data are all computer controlled and the system achieves a complete measurement within 5 min.

Experimentally, we were able to measure $L_{a m b}$ for several types of material such as a-Si:H, Poly-c-Si, $\mathrm{Sb}_{2} \mathrm{~S}_{3}$, etc. Since the sample position is fixed, it can be set into a cryostat, opening the possibility to study materials that have to be kept under vacuum such as organic blends (e.g. P3HT-PCBM) and perovskites [15,17]. Considering the range of $\Lambda$ available, the range of $L_{a m b}$ that can be investigated is of the order of $50-1500 \mathrm{~nm}$. Hence, such a system can be used for routine characterization for laboratories and even for the photovoltaic thin film industry on a large variety of materials.

\subsection{The Fourier transform photocurrent spectroscopy}

Optical absorption below the absorption edge can give fundamental information on the band gap DOS. Indeed, the absorption is linked to the transitions of carriers from occupied states toward empty ones. Therefore, the precise determination of the absorption coefficient $\alpha$ at low energies of photons should bring useful information on the defects of the studied material. Several techniques have been developed to do so among which the constant photocurrent method (CPM), first proposed by Grimmeis and Lebedo [18], was widely applied to thin film semiconductors (a-Si:H, $\mu \mathrm{c}-\mathrm{Si}$, etc.) by M. Vanecek and coworkers [6].

During CPM measurements the sample is enlighten by a monochromatic light and the incident flux $F$ is varied when the photon energy $h v$ is changed to maintain the photocurrent constant on the whole range of explored wavelengths. Assuming that the electrons are the majority carriers, it can be easily shown that an expression of the photoconductivity is

$$
\sigma_{p h}=q \eta \mu_{n} \tau_{n}(1-R) F \alpha,
$$

where $q$ is the absolute charge of the electrons, $\mu_{n}$ their extended state mobility, $\tau_{n}$ their lifetime, $\eta$ the quantum efficiency and $R$ the reflection coefficient of the film. The parameters $\eta, R$ and $\mu_{n}$ are only weakly dependent on the photon energy [19] and it is assumed that, the photocurrent being constant, the splitting of the quasi Fermi levels stays constant during the experiment. Consequently, the recombination and the lifetime $\tau_{n}$ remain constant and the variations of $\alpha_{C P M}$ can be deduced from equation (5) as

$$
\alpha_{C P M}=\frac{K}{F(h v)}
$$

where $K$ is a constant.

However, though CPM brings valuable information, it presents a main drawback to be used for systematic characterization. The adjustment of the flux to maintain the photocurrent constant is time consuming and it may take a few hours to record a complete spectrum from say $0.8 \mathrm{eV}$ to $2 \mathrm{eV}(1550-620 \mathrm{~nm})$. In addition, the wavelength 


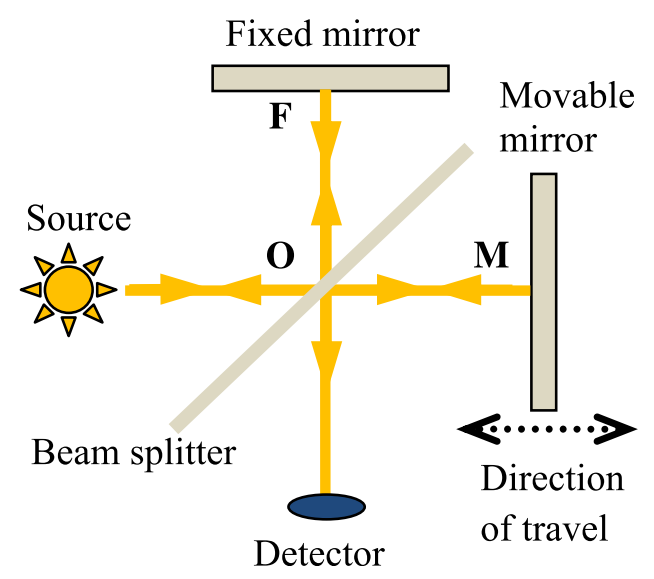

Fig. 2. Schematic diagram of a Michelson interferometer.

resolution of the spectrum is limited by the resolution of the monochromator and, currently, the wavelength step is chosen of the order of $10 \mathrm{~nm}$. To overcome these two issues Poruba and Vanecek have proposed to use the light delivered by an FTIR spectrometer and to treat the sample response with Fast Fourier Transform (FFT) [4,5]. This new technique, the Fourier Transform Photocurrent spectroscopy (FTPS), based on the work of other groups $[20,21]$, proved to be very efficient on $\mu c-S i$ thin films and devices as well as on organic thin films [22]. Since then a detailed overview of the technique has been proposed by J. Holovsky [23] and we present in the following the bases of this Fourier transform analysis.

\subsubsection{FTPS bases}

The FTPS technique using an FTIR spectrometer is much less intuitive than dispersive spectroscopy. In an FTIR spectrometer, instead of a monochromatic beam, a broadband light beam containing many wavelengths is emitted from the output of a Michelson interferometer, a schematic diagram of which is presented in Figure 2. The light source is a collimated halogen lamp illuminating a beam splitter. Part of the light is reflected toward a fixed mirror $\mathbf{F}$ or transmitted toward a movable mirror $\mathbf{M}$ perpendicular to the fixed one. The reflected light on each mirror is sent back toward the beam splitter and transmitted through it, when coming from the fixed mirror, or reflected onto it when coming from the movable mirror, to be sent onto a detector positioned on an axis perpendicular to the axis of the incoming light.

Assuming that the beam splitter is perfect $(50 \%$ transmission and $50 \%$ reflection) the intensity of the light reaching the detector depends on the wavelength and on the differences of optical paths between point $\mathrm{O}$ and the two mirrors $\mathbf{F}$ or $\mathbf{M}: \delta=2(\mathrm{OF}-\mathrm{OM})$. If we call $\omega$ the wavenumber associated to a given wavelength, $\omega=1 / \lambda$, the global response of the detector as function of $\delta$ can be written as [23]

$$
S(\delta)=\int_{-\infty}^{+\infty} B(\omega) R(\omega) \cos (2 \pi \omega \delta) d \omega
$$

where $R$ is the response of the detector as function of $\omega$. The Michelson optical properties may depend on $\omega$ as well as the electronics placed after the detector to record its response. That is why the 'transfer function' of the system $B(\omega)$ is introduced in the expression of $S(\delta)$.

The spectrum $B(\omega) R(\omega)$ can be obtained by an inverse Fourier transform to end with

$$
B(\omega) R(\omega)=\int_{-\infty}^{+\infty} S(\delta) \cos (2 \pi \omega \delta) d \delta
$$

In practice, the detector is replaced by the sample to be studied and the signal coming out of it is treated by the FTIR with fast Fourier transform (FFT). This FFT takes into account the facts that, practically, the integral in equation (7) has finite limits and that the data to treat is obtained by sampling the sample response.

Another point that has to be underlined is that the light reaching the sample is not steady. Indeed, the moving mirror is translated at a constant speed $V$ from which is defined for each $\omega$, the Fourier frequency $f(\omega)$ by

$$
f(\omega)=2 V \omega
$$

For instance, with a speed $V=0.16 \mathrm{~cm} / \mathrm{s}, f(\omega)$ at $400 \mathrm{~nm}(1600 \mathrm{~nm})$ equals $8 \mathrm{kHz}(2 \mathrm{kHz})$.

The FFT being applied to voltage data, an $I-V$ converter is placed after the sample that also amplifies the signal subsequently treated by the FTIR. The bandwidth of the $I-V$ amplifier must be chosen as larger as possible than the maximum Fourier frequency to avoid a modification by the amplifier of the signal coming out of the sample. In addition, the noise inherent to the amplifier must be removed by recording a baseline when no light is sent to the sample, but even after this subtraction the final spectrum contains the term $B(\omega)$ which is unknown

Nevertheless, the sample can be replaced by a reference photodiode, the response of which is known, and by performing the same measurement on this photodiode one can normalize the sample response to that of the photodiode, this normalization being independent of the global transfer function $B(\omega)$

$$
\frac{B(\omega) R_{\text {sample }}(\omega)}{B(\omega) R_{\text {ref }}(\omega)}=\frac{R_{\text {sample }}(\omega)}{R_{\text {ref }}(\omega)} .
$$

Equation (10) is the FTPS equivalent of equation (6) and the relative variations of $\alpha(h \nu)$ can be deduced from it.

The last point to address is the FTIR measurements resolution. The $\omega$ spacing when sampling the film response is usually $4 \mathrm{~cm}^{-1}$. For large wavelengths, say around $1.8 \mu \mathrm{m}$, the wavelength resolution will be of the order of $1.3 \mathrm{~nm}$. At the other end of the spectrum, say around $400 \mathrm{~nm}$, the wavelength resolution will be of the order of $0.06 \mathrm{~nm}$. Hence, even with a standard $\omega$ spacing the resolution is largely better than with a dispersive measurement using a monochromator with a wavelength spacing of $10 \mathrm{~nm}$.

In addition to this high resolution, the fact that at a speed of $V=0.16 \mathrm{~cm}^{-1}$ it takes $1 \mathrm{~s}$ to record a spectrum makes the FTPS a very fast technique even if one averages 


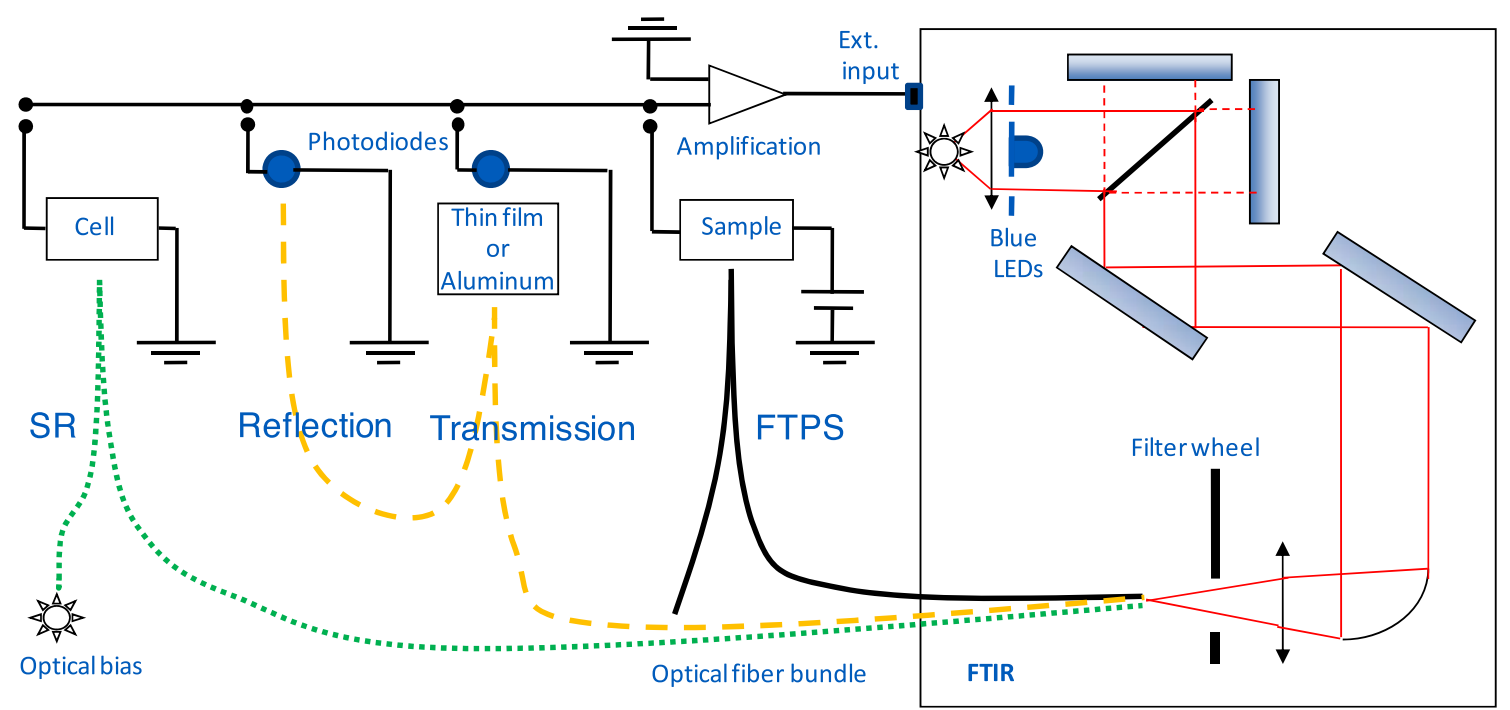

Fig. 3. Schematic diagram of a versatile FTPS set-up.

the data 60 times to improve the signal to noise ratio, and that can be used as a systematic characterization technique for the optimization of photoconductive materials and devices.

\subsubsection{The FTPS bench}

Different schematic diagrams of FTPS benches can be found in references $[5,23,24]$. These benches have the main drawback to be very rigid since the optical components (e.g. parabolic mirror) used to shine light onto the film as well as the sample itself are fixed.

That is why we have built a set-up the diagram of which is displayed in Figure 3. This set-up allows three types of characterization with only very simple adjustments. Details of this bench can be found in [25]. To work in the visible part of the spectrum all the FTIR mirrors are covered with aluminum and the beam splitter of the Michelson interferometer is a quartz plate. The light source is a halogen lamp to which we can add a LED source composed of five LEDs at 405, 420, 450, 470 and $532 \mathrm{~nm}$. With this system we can cover easily a wavelength range from 390 to $1800 \mathrm{~nm}$. In the sample compartment of the FTIR we have installed one branch of a bifurcated silica optical fiber bundle and concentrated the light exiting the Michelson interferometer with a silica lens onto the input of this branch. This latter is set on a XYZ mount to optimize the light collection. A filter wheel is inserted in between the lens and the input of the optical fiber to select ranges of light energies if needed. The advantage of this system is that the output of the optical fiber can be moved in front of any type or size of sample or device fixed on a substrate holder in the air or in a cryostat. The response of the sample is amplified by a low noise and large bandwidth $(50 \mathrm{kHz}$ at a gain of $\left.10^{7}\right) I-V$ amplifier before being sent to an external input of the FTIR to be treated by FFT. To measure the light intensity impinging or going through the films we use two $1 \mathrm{~cm}^{2}$ calibrated photodiodes: a crystalline $\mathrm{Si}$ and a crystalline Ge photodiode, in the $390-1100 \mathrm{~nm}$ and in the 800-1800 nm ranges, respectively.
As mentioned, three different characterizations can be done with this system. For the FTPS the sample is a coplanar film deposited onto a transparent substrate. The sample is fixed onto an electronic circuit plate by two springs that both maintain it mechanically and connect the electrodes to the amplifier and a bias source. A small slit, $0.8 \mathrm{~mm}$ width and $5 \mathrm{~mm}$ height, was drilled in the substrate holder with which it is possible to measure the flux of the light shone onto the sample using the substrate holder alone and placing a calibrated photodiode immediately behind the substrate. It is also possible to measure the flux of light going through the film once fixed onto the substrate holder, the slit being aligned with the electrode spacing. Thus, the FTPS spectra can be deduced from the response of the sample normalized according to equation (10) with the flux of light impinging the sample or with the flux of light going through the film, and the same procedure as for the 'absolute CPM' [26] can be followed to achieve an 'absolute FTPS' measurement.

Transmittance/reflectance measurements can also be achieved on thin films without any electrodes. The fiber bundle being bifurcated, it is possible to record the reflectance by collecting the light reflected by the film and transmitted by the second branch of the bundle and calibrating it replacing the film by an aluminum mirror, the reflectance of which has been calibrated. From these reflection/transmission measurements and from the theoretical developments proposed by Poruba et al. [27], it is possible to deduce optical parameters of the film such as the index, the thickness and $\alpha(h \nu)$ in the high photon energy region, using this latter to calibrate the FTPS spectra to their absolute value as for CPM.

Finally, spectral responses of devices, integrating as absorbers the same films as the ones studied by FTPS, can be performed replacing the coplanar film by the corresponding device. In the case of tandem cells it is possible to use the second branch of the fiber bundle to add a bias of light to the FTIR light. We will not detail this option of the bench we have designed because it is beyond the scope of this paper. 


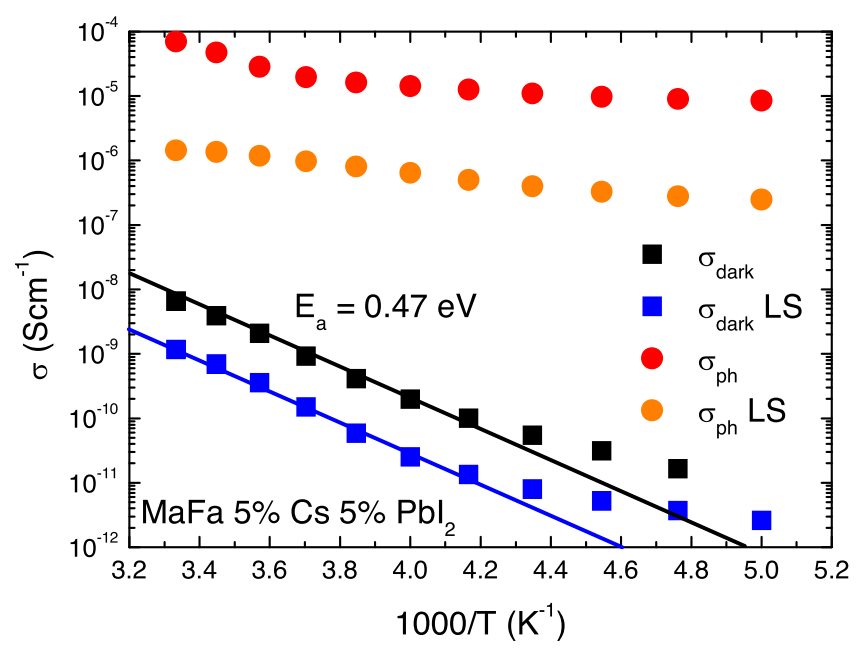

Fig. 4. Arrhenius plots of the dark conductivity (squares) and photoconductivity (circles) of a PVK thin film in its AD and LS states.

\section{Experimental results}

\subsection{Dark photoconductivity and SSPC results}

Dark conductivity and photoconductivity experiments were performed as function of temperature under vacuum $\left(\sim 10^{-6} \mathrm{mBar}\right)$ with an applied voltage in between the electrodes in the range $20-80 \mathrm{~V}$ (20 V for the as-deposited film and $80 \mathrm{~V}$ after light-soaking). The ohmicity of the electrodes was checked before the measurements. The photoconductivity was measured with the same bias and a flux of red light $(633 \mathrm{~nm})$ of the order of $4 \times 10^{16} \mathrm{~cm}^{-2} \mathrm{~s}^{-1}$, as the ones used for SSPG. The temperature was varied from $300 \mathrm{~K}$ to $200 \mathrm{~K}$ in $10 \mathrm{~K}$ steps.

We present in Figure 4 the Arrhenius plots of the dark conductivity, $\sigma_{d a r k}$, and photoconductivity, $\sigma_{p h}$, of a MaFaCs PVK thin film with 5\% Cs and an excess of 5\% $\mathrm{PbI}_{2}$, the sample being in its AD state and LS state. From the dark conductivity plot we deduce an activation energy $E_{a}=0.47 \mathrm{eV}$. This activation energy is not modified by the light-soaking that results in a large decrease of the dark conductivity by a factor of $\sim 7$ and an even larger decrease of the photoconductivity by a factor of $\sim 25$. Light-soaking clearly results in a decrease of the transport properties of the PVK thin film.

\subsection{SSPG results}

We present in Figure 5 typical results obtained on a PVK thin film prepared with $10 \%$ Cs and an excess of $5 \% \mathrm{PbI}_{2}$. In Figure 5a the data (black squares) have been plotted and fitted (red line) according to equation (3), whereas in Figure $5 \mathrm{~b}$ the same data have been plotted and fitted (blue line) according to the Balberg formula (Eq. (4)). One can see that this last plot gives a straight line from which a value of $L_{a m b}=425 \mathrm{~nm}$ is found very close to the value of $410 \mathrm{~nm}$ deduced from the more 'classical' plot.
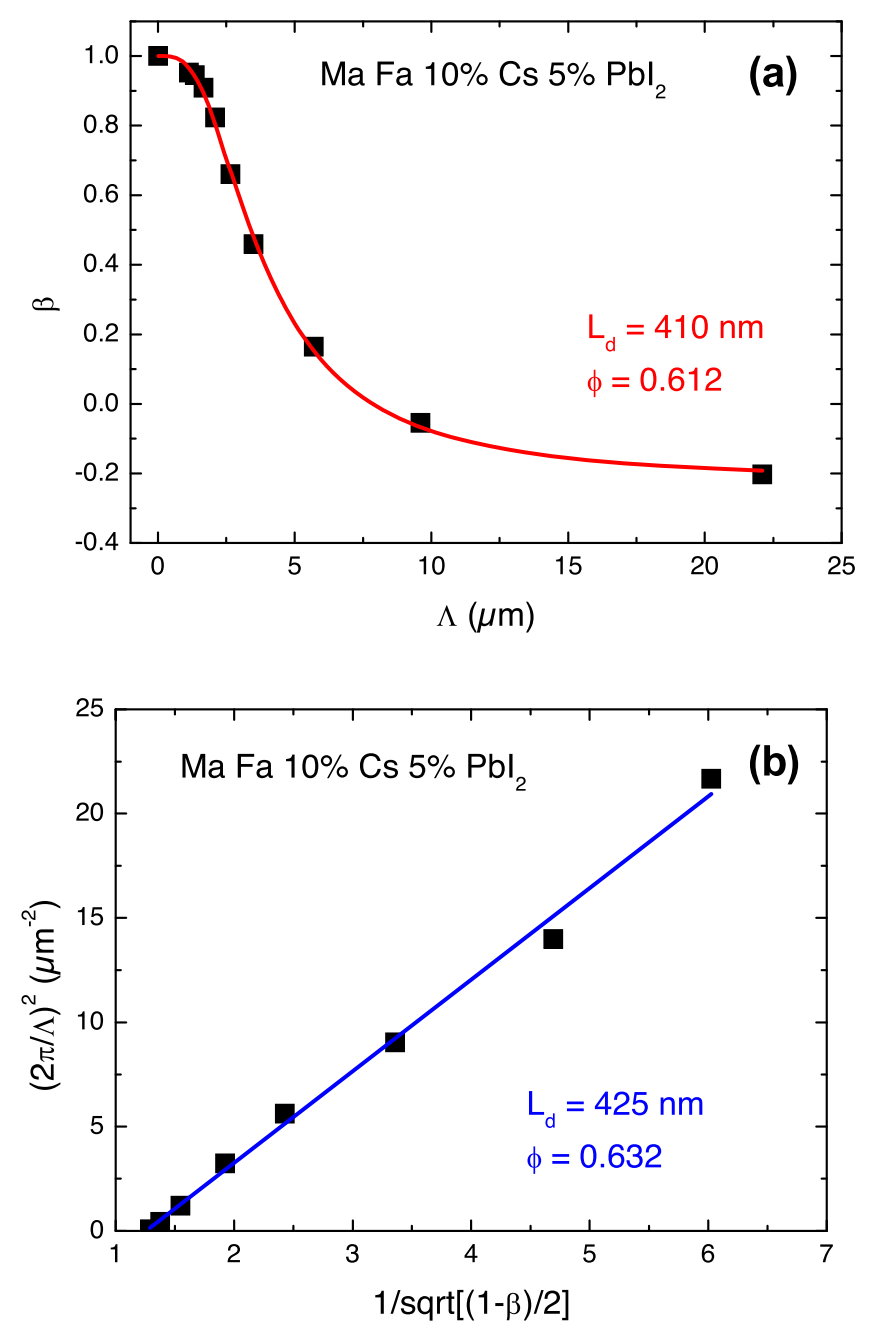

Fig. 5. (a) SSPG data obtained on a PVK film (black squares) fitted with equation (3) (red line), and (b) plotted following the Balberg formula (Eq. (4), black squares) and fitted with a straight blue line, to give an average ambipolar diffusion length of the order of $415 \mathrm{~nm}$.

It must be underlined that with the experimental conditions chosen for the experiment these measurements have been made in the lifetime regime. Indeed, an estimate of the dielectric relaxation time showed that it was around 10 times lower than the carrier lifetime.

For further study, the PVK samples were fixed onto the cold finger of a cryostat and, using the fast automated SSPG bench, we followed the evolution of $L_{a m b}$ with temperature during consecutive annealings of the films. For several samples we have observed a decrease of the ambipolar diffusion length when the films were put under vacuum and annealed, the final values of $L_{a m b}$ being much lower than those measured under air. In addition, the $L_{a m b}$ values seemed to stabilize at these low values even after several subsequent annealings.

An example of the evolution of $L_{a m b}$ with annealing is shown in Figure 6. We have to underline that with some samples we observed a decrease of $L_{a m b}$ even without 


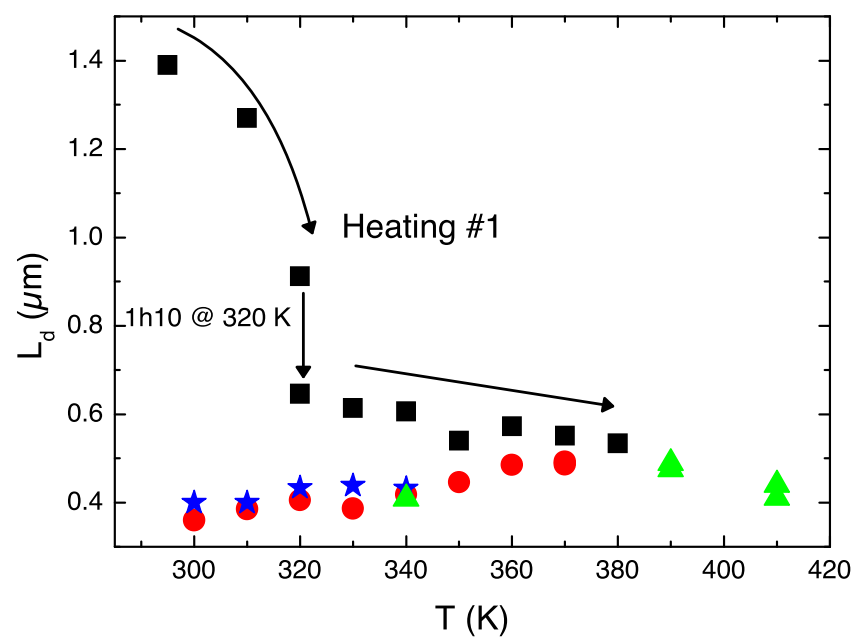

Fig. 6. $L_{a m b}$ evolution with several annealings. Black squares correspond to the first annealing up to $380 \mathrm{~K}$ (arrows indicate the $L_{a m b}$ evolution). Blue stars correspond to the $L_{a m b}$ evolution after this annealing. Red circles give the $L_{a m b}$ evolution after a second annealing at $370 \mathrm{~K}$ during $1 \mathrm{~h} 10$ and the green triangles were obtained after subsequent annealings at 390 and $410 \mathrm{~K}$.

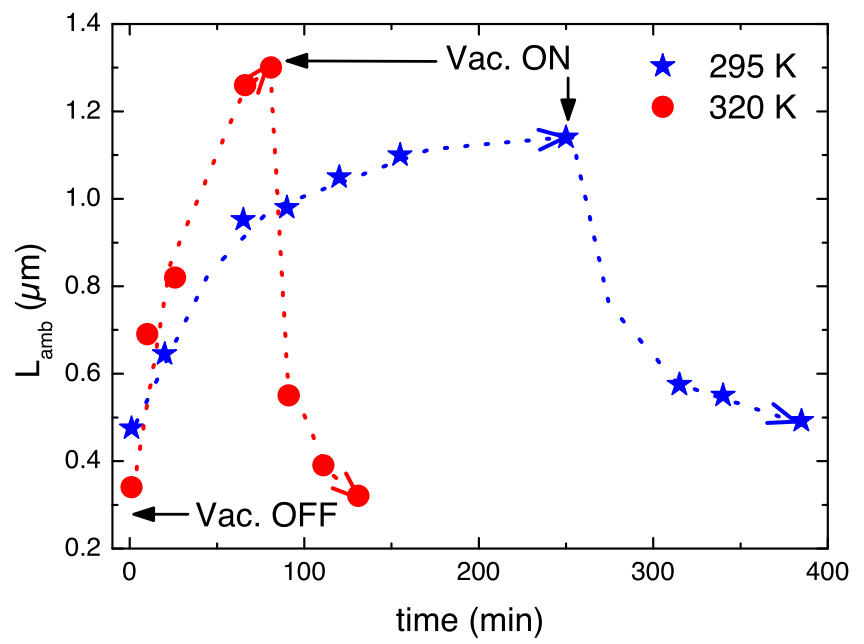

Fig. 7. Evolution of the diffusion lengths measured when the sample is put under air (vac. OFF) or under vacuum (vac. ON) at two temperatures: $295 \mathrm{~K}$ (stars), $320 \mathrm{~K}$ (circles). Dashed arrows are guides to the eye.

annealing, the films being just put under vacuum. Again, for these films the $L_{a m b}$ values stabilized at lower values than those measured under air.

To distinguish between the influence of the environment (air or vacuum) and of the temperature on the $L_{a m b}$ values we have performed experiments on the same film as function of the time under which the film was in air or under vacuum. The results are summarized in Figure 7. Starting from a film hold at $295 \mathrm{~K}$ under vacuum during several hours, we have observed an increase of $L_{a m b}$ when the film was put under air (vac. OFF) followed by a decrease of $L_{a m b}$ when the sample was put back under vacuum (vac. ON). Therefore, the $L_{a m b}$ evolution seems to be essentially linked

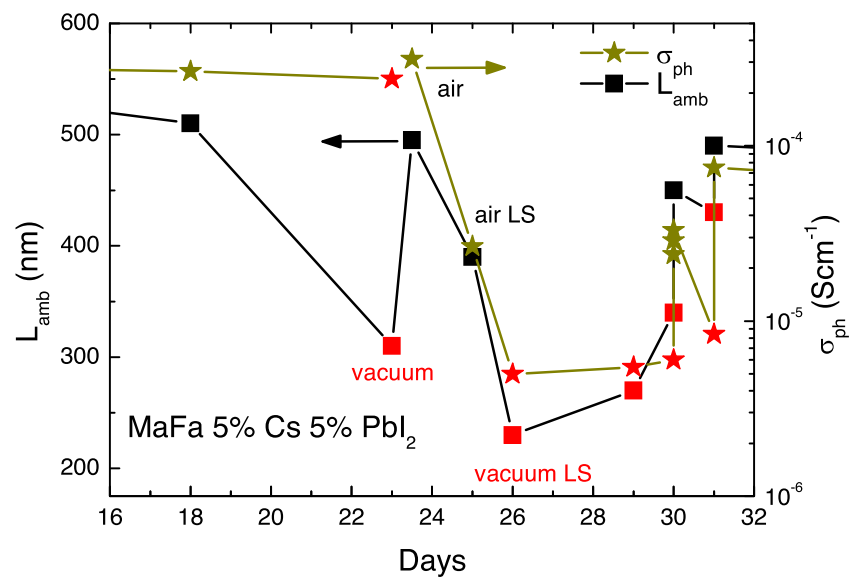

Fig. 8. Evolution of the diffusion length (squares) and of the photoconductivity (stars) of a PVK thin film with its environment (air or vacuum (red symbols)) and with light-soaking. Lines are guides to the eye.

to the film environment, air or vacuum. Nevertheless, this process seems to be activated with temperature, being much faster at $320 \mathrm{~K}$ than at room temperature.

We have also investigated on the evolution of $L_{a m b}$ with light-soaking. The data (squares) combined with measurements of the photoconductivity $\sigma_{p h}$ (stars) are displayed in Figure 8. The SSPG and the SSPC measurements were done with the same DC flux of $F=4 \times 10^{16} \mathrm{~cm}^{-2} \mathrm{~s}^{-1}$. The data values on day 1 (not shown in the figure) were $L_{a m b}=580 \mathrm{~nm}$ and $\sigma_{p h}=3.0 \times 10^{4} \mathrm{Scm}^{-1}$. It can be seen that both $L_{a m b}$ and $\sigma_{p h}$ are systematically lower when measured under vacuum (red symbols) than under air (dark symbols), independently of the state of the sample, $\mathrm{AD}$ and LS.

After light-soaking under air during $45 \mathrm{~h}$ we note a decrease of both $L_{a m b}$ and $\sigma_{p h}$ (air $\rightarrow$ air LS), $L_{a m b}$ by $20 \%$ and $\sigma_{p h}$ by a factor of $\sim 10$. Then, the film being maintained under vacuum during 5 days, its transport properties seem to recover; almost completely for $L_{a m b}$, the values on day 31 being almost the same as on day 23 before light-soaking, and $\sigma_{p h}$ at least partly, its value on day 31 being only 5 times smaller than the value on day 23 . These results show that with light-soaking $L_{a m b}$ for this type of material is rather stable whereas, in the other hand, $\sigma_{p h}$ degrades quite a lot.

Trying to link these behaviors to the density of states of the material we have also performed FTPS characterizations.

\subsection{FTPS results}

The FTPS spectra were recorded by normalizing the sample response to the direct flux impinging the sample according to equation (10).

We present in Figure 9a the influence of light-soaking on a PVK film, the sample being maintained in the air. All the spectra have been normalized to the same value above $1.7 \mathrm{eV}$. It can be seen that there are no differences for the spectrum recorded the sample being in the $\mathrm{AD}$ state (black 

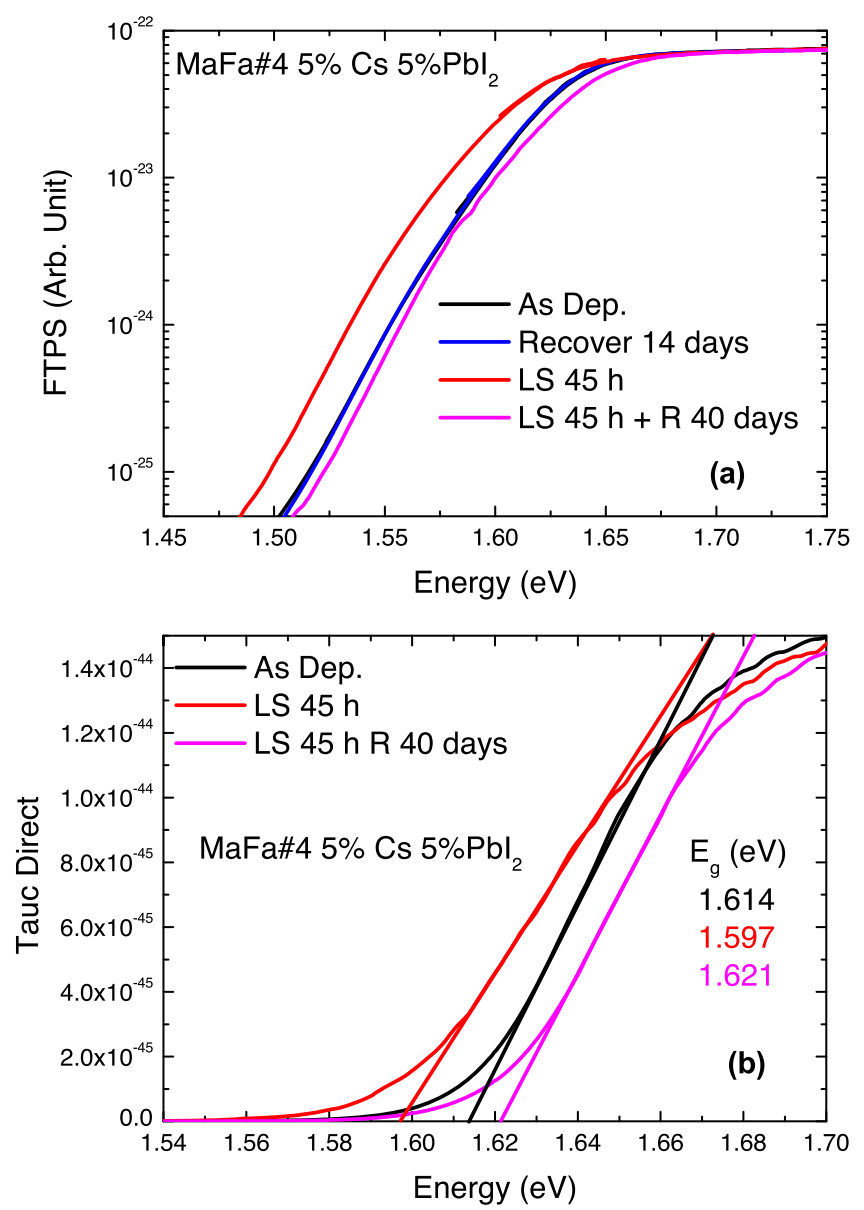

Fig. 9. Evolution with time and light-soaking, (a) of the FTPS spectra, (b) of the Tauc gap of a perovkite thin film.

line) and the same sample maintained under primary vacuum $\left(10^{-1} \mathrm{mBar}\right)$ and in the dark during 14 days (blue line).

After $45 \mathrm{~h}$ of light-soaking the band edge is shifted toward low energy (red line) with no modification of the Urbach energy, $E_{u} \approx 16 \mathrm{meV}$, and after a recovery of 40 days under dark and primary vacuum the band gap has widen again (purple line). It is worth to note that the same type of sample left under air during some 65 hours did not present any evolution of the band gap when tested with the FTPS technique.

To quantify the evolution of the band gap with lightsoaking we have plotted the evolution of the Tauc gap. Assuming that the FTPS data is representative of the variations of the absorption coefficient with energy, the Tauc gap is calculated from the linear extrapolation toward the energy axis of the linear part of the plot of (FTPS data $\times h v)^{2}$ versus $h v$. These plots are presented in Figure $9 \mathrm{~b}$ and a clear evolution of the Tauc gap is observed with a value of $E_{g}=1.614 \mathrm{eV}$ in the $\mathrm{AD}$ state that decreases down to $E_{q}=1.597 \mathrm{eV}$ after LS, and increases up to $E_{g}=1.621 \mathrm{eV}$ after a recovery of 40 days.

This light-soaking experiment was repeated on the same sample with measurements of the FTPS spectra at different time intervals. It appears that the bang gap of the
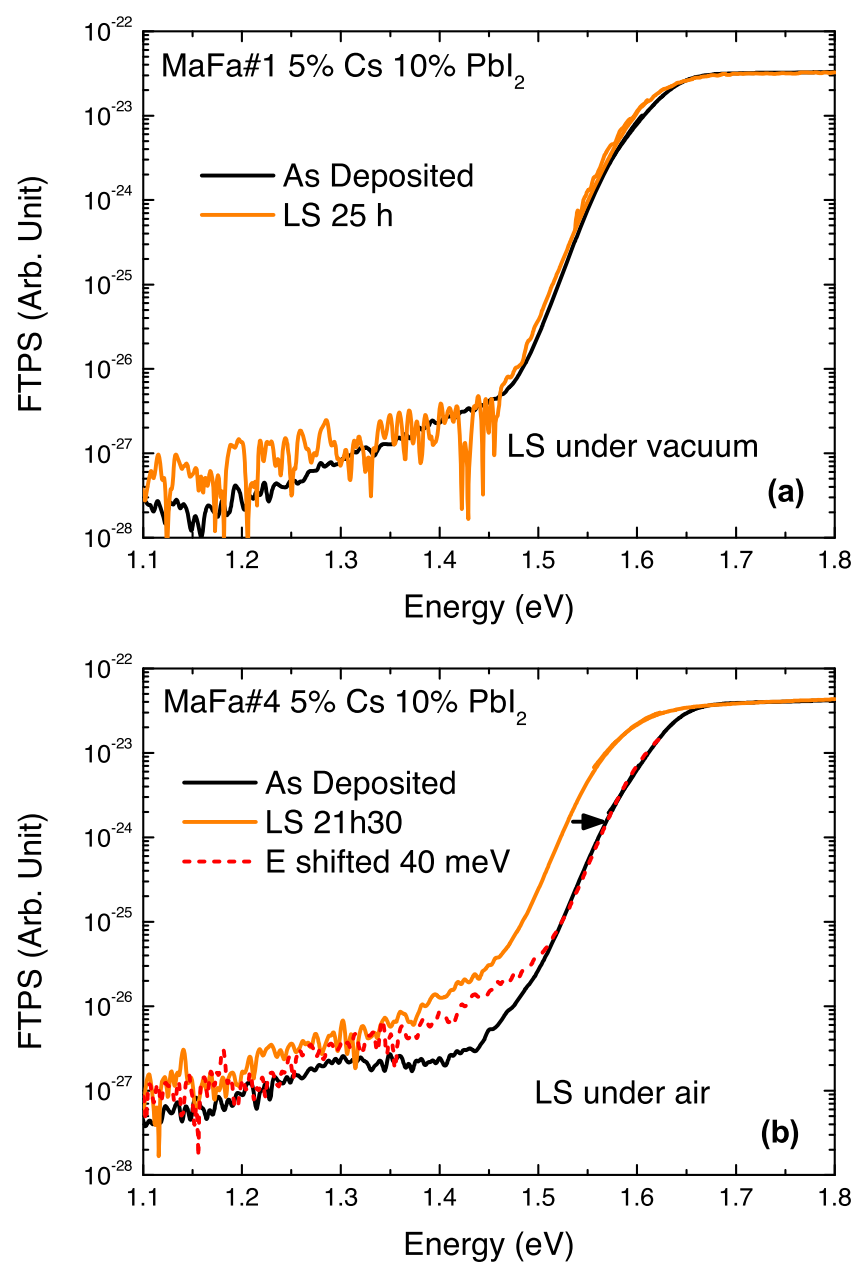

Fig. 10. Evolution of the FTPS spectra of perovskite samples with light-soaking (a) under vacuum, (b) under air, the arrow indicates a shift of $40 \mathrm{meV}$ upward of the LS spectrum.

film is steadily decreasing with the light-soaking time. From the plots of the Tauc gap, we found that the bang gap was shrinking from $1.621 \mathrm{eV}$ down to $1.598 \mathrm{eV}$ from the recovered state (purple line of Fig. 9b) to the new LS state obtained after 21 hours of light-soaking. According to these results, the evolution of the band gap with light-soaking appears to be a reproducible and reversible phenomenon.

Since we have observed a shrinking of the band gap with light-soaking under air and no evolution of the gap under air alone, we have investigated on the influence of the air by achieving a light-soaking under vacuum. Figure 10a shows FTPS spectra obtained on the same film in the AD state and after 25 hours of light-soaking. It can be observed that the gap red-shift is extremely small and that, despite of the noise, the deep states down to $1.3 \mathrm{eV}$ are also very similar. It is difficult to assess that there is an increase of the deep states below $1.3 \mathrm{eV}$, the signal to noise ratio being too low for an accurate measurement. Nevertheless, on the same sample we have observed a decrease of the dark and photoconductivity by a factor $\sim 10$.

On the other hand, a film with the same composition and LS under air during the same amount of time presents, as already seen, a red-shift of its band gap along with a 
slight increase of the deep defect density (see Fig. 10b). This increase of the deep defect density is clearly visible when one shifts upward by $40 \mathrm{meV}$ the FTPS spectrum obtained after light-soaking (dashed red curve): $E_{u}$ is the same but around $1.4 \mathrm{eV}$ an increase of the FTPS spectrum by a factor $3-4$ is observed. Note that such an increase of the deep defect density upon light-soaking was already suggested by W. Tress [28]. The decrease of the film photoconductivity was also of the order of 10 as for the film LS under vacuum.

\section{Discussion}

As far as the PVK thin films we have studied are concerned, the experimental results presented in Section 4 can be summarized as follows:

- air is not systematically detrimental to the transport properties of the PVK films since we have observed a decrease of the transport parameters, $L_{a m b}$ and $\sigma_{p h}$, when the samples were put under vacuum. The evolution of $L_{a m b}$ values between air and vacuum seems to be reversible and activated with temperature,

- light-soaking results in a decrease of the film transport parameters, $L_{a m b}$ and $\sigma_{p h}$, as well as a decrease of the dark conductivity. When performed under air, it also results in a shrinking of the bang gap and a slight increase of the deep defect density, two evolutions that are not observed when light-soaking is achieved under vacuum,

- after light-soaking, the sample being left a few days under dark and under vacuum, some properties seem to recover whereas some others do not. For instance, the red-shift of the gap after light-soaking disappears after a few days of recovery and one can see in Figure 8 that $L_{a m b}$ on day 31, after light-soaking and some days of recovery, is almost equal to the diffusion length on day 23 before lightsoaking. On the other hand, the photoconductivity never recovers the value it had before light-soaking.

This summary indicates that different processes are involved in the evolution of the material properties.

Concerning the increase of $L_{a m b}$ under air compared to $L_{a m b}$ under vacuum, we may suggest that adsorption of an air component (water vapor or oxygen) may passivate the sample surface reducing surface recombination. This assumption is in agreement with previous reports in the literature suggesting that oxygen ingress or moisture incorporation could be responsible for an increase of $L_{a m b}$ [29]. Oxygen influence was also underlined by GalisteoLopez et al. [30] and by Brenes et al. [31] who observed an enhancement of the photoluminescence quantum yield after exposure of the films to air and light. It was proposed that the formation of a surface passivating super-oxide on the film surface was responsible for surface states passivation leading to an increase of the carrier lifetime. Reversible trap passivation was also theoretically predicted and studied by Meggiolaro et al. [32]. In all these publications the passivation of the film surface by an air component was found to be lightassisted. In our case the light is simply provided by the main beam of the SSPG experiment also used in the SSPC measurements.
Concerning the evolution of material characteristics with light-soaking, different processes seem to be involved: an evolution of the band gap, and a degradation of $L_{a m b}$ and of the photoconductivity. These signatures may not have the same origin. Indeed, the band gap evolution of the material seems to be linked to a combined influence of light and air since FTPS spectra of PVK thin films do not present any evolution neither when left under air, even during $65 \mathrm{~h}$, nor when illuminated under vacuum. Evolutions of the FTPS spectra are only seen when the films are light-soaked under air. In addition, after a few days of recovery the band gap width returns to its $\mathrm{AD}$ value.

On the other hand, a decrease of $\sigma_{p h}$ is observed when the film is light-soaked both in air and under vacuum, indicating that this degradation is mainly linked to the influence of light alone. Moreover, even though we observe a small recovery of $\sigma_{p h}$ after a few days under dark and vacuum, the $\sigma_{p h}$ decrease appears irreversible, at least at room temperature.

Evolution of the band gap with light-soaking was also observed by other groups either from small variations of the absorptance spectra [33] or from Photo Deflection Spectroscopy (PDS) [34]. They have attributed these variations to modifications of the surface of the films by creation of low gap regions and trapping states. However, the PDS technique is known to be highly sensitive to surface states [35] whereas the FTPS technique is more sensitive to bulk states. Thus, though we cannot reject some surface modification, as observed in the increase of $L_{a m b}$ when the film is exposed to air and light, the bandgap shrinking is more likely linked to an evolution occurring in the volume of the film. Note that the decrease of the bandgap $(\sim 1.6 \mathrm{eV})$ is of the order of 25 to $40 \mathrm{meV}$, that is around $2 \%$. A possible explanation of the bandgap red-shift is that an expansion of the lattice by the same amount could be responsible for it, since such an expansion was already observed during light-soaking in the case of a-Si:H [36].

It could also explain why we have observed by GD-OES a large diffusion of gold from the back electrode into the film during light-soaking (see Fig. 11). This experiment was performed on three different films prepared at the same time under the same conditions. Almost no diffusion of gold can be noticed for the film kept under its AD state whereas gold is seen to diffuse through the whole thickness of the LS films, the longer the light-soaking time the larger the diffusion.

Gold diffusion, as well as other metals like $\mathrm{Ag}$ and $\mathrm{Cu}$, in the PVK films under light-soaking has been already underlined by other groups $[8,37,38]$, this diffusion being highly detrimental to the device performances.

The fact that we do not observe a gap shrinking when the sample is light-soaked under vacuum does not mean that there is no expansion of the film during this process. It just indicates that when we performed FTPS measurements after light-soaking the lattice was back to its original position. Observing a red-shift of the bandgap when lightsoaking is performed under air suggests that during lightsoaking an air component diffuses inside the film, as the gold, and slows down the return of the lattice to its original position, this return depending on the time needed for this 


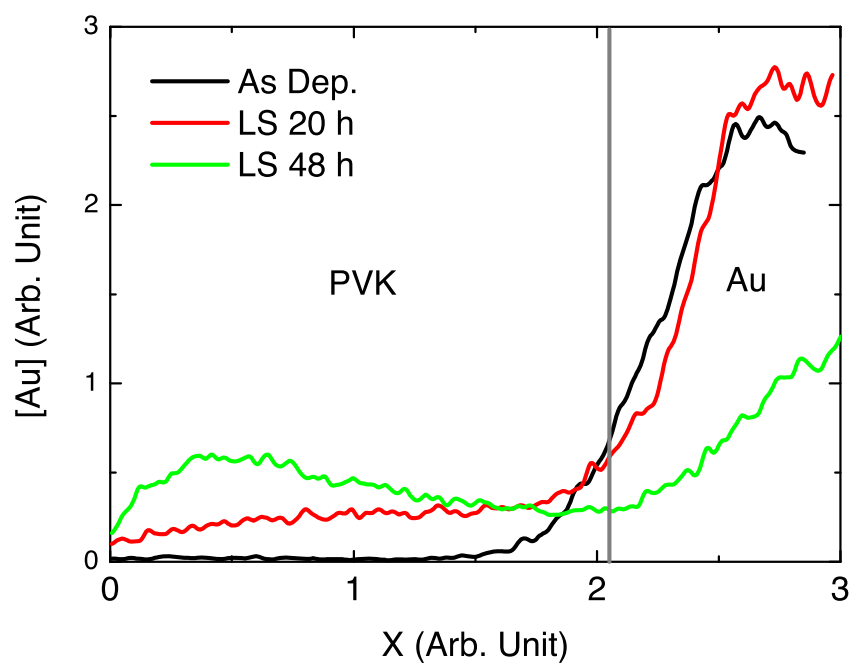

Fig. 11. Gold diffusion through PVK films revealed from GDOES experiments performed on different films: one in its AD state (black line), LS during $20 \mathrm{~h}$ (red line) and $48 \mathrm{~h}$ (green line). The vertical grey line indicates the border between the PVK films and the gold electrode.

air component to desorpt. That is why, after light-soaking under air, a few days under vacuum are necessary for the bandgap to return to its original value.

The last point to address is the decrease of $\sigma_{p h}$ resulting from light-soaking. This decrease seems to be irreversible, at least at room temperature, in contradiction with what we observed with the ambipolar diffusion length. Some authors have suggested that light-soaking resulted mostly in a decrease of the carrier lifetime though, in some cases, a decrease of the mobility was also proposed [33]. A decrease of the carrier lifetime can be due to an increase of the recombining states or an increase of their capture cross-section or both. However, from the FTPS spectra we have not observed a large increase of the DOS. For the light-soaking under air we found a slight increase by a factor of 3 to 4 and no increase at all with light-soaking under vacuum. In the mean time $\sigma_{p h}$ decreases by a factor of 5 to 10 whereas $L_{a m b}$ is almost unaffected.

The mobility $\times$ lifetime products, $\mu \tau$, can be deduced from the experimental data and equation (5) for $\sigma_{p h}$ or from equation (9) of reference [12] for $L_{a m b}$. Before light-soaking, one finds $\mu \tau_{\sigma} \approx 2 \times 10^{-6}$ and $\mu \tau_{L} \approx 5 \times 10^{-8} \mathrm{~cm}^{2} / \mathrm{V}$, and after light-soaking we have $\mu \tau_{\sigma} \approx 5 \times 10^{-7}$ and $\mu \tau_{L} \approx$ $5 \times 10^{-8} \mathrm{~cm}^{2} / \mathrm{V}$. We always have $\mu \tau_{\sigma} \gg \mu \tau_{L}$ which indicates that transport properties depend on majority and minority carriers. If a new recombination path via gap states had been opened or enhanced by light-soaking it is highly probable that both quantities $\sigma_{p h}$ and $L_{a m b}$ would have been affected. Since it is not the case, this result suggests that the decrease of the majority carrier $\mu \tau$ product could be essentially due to a decrease of their mobility instead of a decrease of their lifetime. The reader may note that such a decrease of mobility has been already observed [33]. Though it concerned films with higher bromide contents than ours, a decrease of the majority carrier mobility can be considered as possible explanation of the photoconductivity decrease under light-soaking.

\section{Conclusions}

We have developed fast and efficient characterization techniques the application of which allowed following the evolution of transport properties of PVK thin films placed in different environments and light-soaked.

With these techniques we have found that air is not systematically detrimental to the PVK transport properties, since we observed increases of the diffusion length and photoconductivity when samples were exposed to air.

We have also put into evidence that the diffusion length and the density of states are only little affected by lightsoaking indicating a rather good stability of the triple cations PVK films. Nevertheless, light-soaking induces a decrease of the photoconductivity by a factor of the order of five to ten. It could be one of the reasons why the conversion efficiency of devices decreases when exposed to 1 sun illumination. However, the diffusion of gold from the electrodes we have observed under light-soaking is probably much more detrimental to the device conversion efficiency than the small degradations of the transport properties. Reaching a better stability of devices seems to imply a better control of the influence and evolution of the electrodes under illumination.

We acknowledge the help of A. Rebai and H.-J Lin for PVK thin film deposition. We are grateful to M. Police and R. Andlauer for technical assistance to develop the SSPG and FTPS benches. The GD-OES experiments were performed by S. Gaiaschi from Horiba-Jobin\&Yvon. Many thanks to her.

\section{References}

1. A. Kojima, K. Teshima, Y. Shirai, T. Miyasaka, J. Am. Chem. Soc. 131, 6050 (2009)

2. D. Ritter, E. Zeldov, K. Weiser, Appl. Phys. Lett. 49, 791 (1986)

3. C. Longeaud, Rev. Sci. Instrum. 84, 055101 (2013)

4. A. Poruba, M. Vanecek, J. Rosa, L. Feitknecht, N. Wyrsch, J. Meier, A. Shah, T. Repmann, B. Rech, in Proceedings of the 17th European Photovoltaic Solar Energy Conference, 2001, (WIP, Munich, Germany), p. 2981

5. M. Vaněček, A. Poruba, Appl. Phys. Lett. 80, 719 (2002)

6. M. Vaněček, J. Kočka, J. Stuchlík, A. Tříska, Solid State Commun. 39, 1199 (1981)

7. W. Grimm, Spectrochim. Acta B 23, 443 (1968)

8. S. Cacovich, L. Ciná, F. Matteocci, G. Divitini, P.A. Midgley, A. Di Carlo, C. Ducati, Nanoscale 9, 4700 (2017)

9. D. Ritter, K. Weiser, E. Zeldov, J. Appl. Phys. 62, 4563 (1987)

10. I. Balberg, A.E. Delahoy, H.A. Weakliem, Appl. Phys. Lett. 53, 992 (1988)

11. E. Sauvain, J.H. Chen, J. Appl. Phys. 75, 5191 (1994)

12. R. Brüggemann, J. Phys.: Conf. Ser. 253, 012081 (2010) 
13. R. Brüggemann, in Advanced Characterization Techniques for Thin Film Solar Cells, Second Edition, edited by D. Abou-Ras, T. Kirchartz, U. Rau (Wiley-VCH Verlag, 2011), p. 163

14. C.-D. Abel, G.H. Bauer, W.H. Bloss, Philos. Mag. B 72, 551 (1995)

15. C. Longeaud, A. Fath Allah, J.A. Schmidt, M. El Yaakoubi, S. Berson, N. Lemaitre, Org. Electr. 31, 253 (2016)

16. I. Balberg, Phys. Rev. B 44, 1628 (1991)

17. C. Longeaud, F.J. Ramos, A. Rebai, J. Rousset, Sol. RRL 2, 1800192 (2018)

18. H.G. Grimmeiss, L.A. Lebedo, J. Appl. Phys. 46, 2155 (1975)

19. J. Kocka, M. Vanecek, A. Triska, in Advances in Amorphous Semiconductors, edited by H. Fritzsche (World Scientific Publisher, Singapore, 1989)

20. J.W. Tomm, A. Jaeger, A. Bärwolff, T. Elsaesser, A. Gerhardt, J. Donecker, Appl. Phys. Lett. 71, 2233 (1997)

21. T. Inushima, M.H. Brodsky, J. Kanicky, R.J. Serino, in Proceedings of the AIP Conference, 1984 (AIP, New York, USA), Vol. 120, p. 24

22. L. Goris, A. Poruba, L. Hod'ákova, M. Vaněček, K. Haenen, M. Nesládek, P. Wagner, D. Vanderzande, L. De Schepper, J. V. Manca, Appl. Phys. Lett. 88, 052113 (2006)

23. J. Holovsky, in Fourier Transforms-New Analytical Approaches and FTIR Strategies, edited by G. Nikolic (IntechOpen, 2011), Chap. 13

24. A. Poruba, M. Vanecek, J. Meier, A. Shah, J. Non-Cryst. Solids 299-302, 536 (2002)

25. N. Puspitosari, C. Longeaud, Rev. Sci. Instrum. 88, 08611 (2017)

26. M. Vanecek, J. Kocka, A. Poruba, A. Fejfar, J. Appl. Phys. 78, $6203(1995)$

27. A. Poruba, A. Fejfar, Z. Remeš, J. Špringer, M. Vanecek, J. Kocka, J. Meier, P. Torres, A. Shah, J. Appl. Phys. 88, 148 (2000)
28. W. Tress, M. Yavari, K. Domanski, P. Yadav, B. Niesen, J.P. Corea Baena, A. Hagfeldt, M. Graetzel, Energy Environ. Sci. 11, 151 (2018)

29. S. De Wolf, J. Holovsky, S.-J. Moon, P. Löper, B. Niesen, M. Ledinsky, F.-J. Haug, J.-H. Yum, C. Ballif, J. Phys. Chem. Lett. 5, 1035 (2014)

30. J.F. Galisteo-Lopez, M. Anaya, M.E. Calvo, H. Miguez, J. Phys. Chem. Lett. 6, 2200 (2015)

31. R. Brenes, D. Guo, A. Osherov, N.K. Noel, C. Eames, E.M. Hutter, S.K. Pathak, F. Niroui, R.H. Friend, M.S. Islam, H.J. Snaith, V. Bulović, T.J. Savenije, S.D. Stranks, Joule 1, 155 (2017)

32. D. Meggiolaro, E. Mosconi, F. De Angelis, ACS Energy Lett. 2, 2794 (2017)

33. D. Guo, Z. Andaji-Garmaroudi, M. Abdi-Jalebi, S.D. Stranks, T.J. Savenije, ACS Energy Lett. 4, 2360 (2019)

34. Z. Andaji-Garmaroudi, M. Abdi-Jalebi, D. Guo, S. MacPherson, A. Sadhanala, E.M. Tennyson, E. Ruggeri, M. Anaya, K. Galkowski, R. Shivanna, K. Lohmann, K. Frohna, S. Mackowski, T.J. Savenije, R.H. Friend, S.D. Stranks, Adv. Mater. 31, e1902374 (2019)

35. Z.E. Smith, V. Chu, K. Shepard, S. Aljishi, D. Slobodin, J. Kolodzey, S. Wagner, T.L. Chu, Appl. Phys. Lett. 50, 1521 (1987)

36. T. Gotoh, S. Nonomura, M. Nishio, N. Msui, S. Nitta, M. Kondo, A. Matsuda, J. Non-Cryst. Solids 227-230, 263 (1998)

37. C.C. Boyd, R. Cheacharoen, K.A. Bush, R. Prasanna, T. Leijtens, M.D. McGehee, ACS Energy Lett. 3, 1772 (2018)

38. K. Domanski, J-P. Correa-Banea, N. Mine, M. Khaja Nazeeruddin, A. Abate, M. Saliba, W. Tress, A. Hagfeldt, M. Grätzel, ACS Nano 10, 6306 (2016)

Cite this article as: Christophe Longeaud, Study of transport parameters and defect states in thin film perovskites under different environments - air or vacuum - and after light-soaking, EPJ Photovoltaics 11, 5 (2020) 